\section{Validity and reliability of three-dimensional scanning compared to conventional anthropometry for children and adolescents: methodological mistake}

Advance online publication, 31 May 2017; doi:10.1038/pr.2017.73

To the Editor: I read with interest the paper by Glock F et al. (1) published in the January 2017 edition of Pediatric Research. To use three-dimensional whole-body laser scanning in routine clinical practice, validity and reliability will have to be confirmed. The purpose of the authors was to compare a whole-body laser scanning with conventional anthropometry in a group of 473 children and adolescents from the Leipzig Research Centre for Civilization Diseases (LIFE-Child) (1). Concordance correlation coefficients (CCCs) were calculated to assess validity. Overall CCCs (OCCCs) were used to analyze intraobserver reliability (1).

Reproducibility (precision) and validity (accuracy), as two completely different methodological issues, should be assessed using appropriate tests. Regarding reliability, for quantitative variables intraclass correlation coefficient (ICC) and for qualitative variables weighted kappa should be used with caution. However, to assess validity, for quantitative variables interclass correlation coefficient (Pearson $r$ ) and for qualitative variables sensitivity, specificity,)positive predictive value, negative predictive value, likelihood ratio positive and likelihood ratio negative, as well as diagnostic accuracy and odds ratio, are among the most appropriate tests $(2-8)$.

Why did the authors not use the abovementioned wellknown estimates to assess reliability and validity?

Moreover, for reliability analysis, an individual-based approach should be applied using ICC agreement in single measure because approaching a global average (OCCC) can simply yield misleading messages. The concordance cell can be exactly the same, with no reliability at all. Finally, to correctly assess reliability, not only should both concordance and discordance cell be considered, but also the number of categories as well as prevalence of the concordance cells should also be taken into account (2-8).

Based on the author's results, the intraobserver reliability of both techniques is "excellent" (OCCC $\geqslant 0.9$ ). At the same time, neck and thigh circumference showed a "good" $(C C C \geqslant 0.7)$ and head circumference a "low" (CCC $<0.5$; flipping a coin!) degree of concordance over the complete study population (1). They thus concluded that scanning can be used in an epidemiologic setting with children and adolescents, but some measurements should be considered with caution due to reduced agreement with conventional anthropometry (1). Such conclusion may be misleading because of not taking into account the abovementioned statistical and methodological issues. Therefore, in routine clinical practice, misdiagnosis and mismanagement of the patients cannot be avoided.

\section{Siamak Sabour ${ }^{1}$ \\ ${ }^{1}$ Safety Promotion and Injury Prevention Research Center, Shahid Beheshti University of Medical Sciences, Tehran, I.R. Iran.}

Correspondence: Siamak Sabour, s.sabour@sbmu.ac.ir

\section{REFERENCES}

1. Glock F, Vogel M, Naumann S, et al. Validity and intra-observer reliability of three-dimensional scanning compared to conventional anthropometry for children and adolescents from a population-based cohort study. Pediatr Res 2017;81:736-44.

2. Sabour S, Ghassemi F. Reliability of the International Spinal Cord Injury Musculoskeletal Basic Data Set; methodological and statistical issue to avoid misinterpretation. Spinal Cord Ser Cases 2016;2:16023.

3. Sabour S, Ghassemi F. Accuracy and reproducibility of the ETDRS visual acuity chart: methodological issues. Graefes Arch Clin Exp Ophthalmol 2016;254:2073-74.

4. Sabour S. Reliability of a new modified tear breakup time method: methodological and statistical issues. Graefes Arch Clin Exp Ophthalmol 2016;254:595-96.

5. Sabour S. Adherence to guidelines strongly improves reproducibility of brachial artery flow-mediated dilation. Common mistakes and methodological issue.. Atherosclerosis 2016;251:490-91.

6. Sabour S. Re: does the experience level of the radiologist, assessment in consensus, or the addition of the abduction and external rotation view improve the diagnostic reproducibility and accuracy of MRA of the shoulder? Clin Radiol 2015;70:333-4.

7. Sabour S. Reliability of the ASA physical status scale in clinical practice: methodological issues. Br J Anaesth 2015;114:162-63.

8. Sabour S. Validity and reliability of the robotic objective structured assessment of technical skills. Obstet Gynecol 2014;124:839. 